\title{
CLEAN/MEM DECONVOLUTION ERRORS: SEMICOMPACT SOURCES
}

\author{
D. S. BRIGGS* \\ ATNF, PO Box 76, Epping, NSW 2121, Australia \\ and \\ T. J. CORNWELL \\ NRAO, PO Box O, Socorro, NM 87801, USA
}

\begin{abstract}
We have run a number of simulations investigating the limitations of noiseless point spread deconvolution with the VLA. On-source errors of more than .01 and off-source dynamic ranges of less than 15,000 were found for some parameters typical of high precision observations. Deconvolution errors were not confined to the support of the source, and limit the dynamic range in at least one VLA observation.
\end{abstract}

Key words: Deconvolution - CLEAN - MEM

\section{Introduction}

The initial impetus for this investigation came from an observation of $3 \mathrm{C} 48$ that was unaccountably limited to a dynamic range of some $16000: 1$, a factor of 10 above the thermal noise. A simple image plane model of the source was constructed and the deconvolution process simulated. The venerable Högbom CLEAN, (Högbom 1974), produced a dynamic range of 26000:1 on a noiseless, perfectly calibrated realization of this model, providing for the first time evidence that the deconvolution process was a limiting factor comparable to residual calibration errors for high precision VLA observations. Moreover, the morphology of the error pattern closely matched that seen in the observational map, although reduced in amplitude. Subsequent work brought the agreement between simulated and measured RMS error to within $2 \%$ ! At the observation frequency of $8.4 \mathrm{GHz}$, and using the VLA's high resolution A configuration, $3 \mathrm{C} 48$ is a semicompact source, with two nearly unresolved components and a modest extended halo. This is close to an ideal source for CLEAN, so its failure was surprising. Other deconvolution algorithms, including the MEM implementation VM (Cornwell \& Evans 1985), SDI-CLEAN (Steer, Dewdney, \& Ito 1984) and Multi-Resolution CLEAN (Wakker \& Sch.varz 1988) fared little better. Eventually it was discovered that a similar error mo: phology was produced with a single isolated Gaussian model, and a set of simulations was run against the width of the model source. The reconvolution beamwidth proved a crucial parameter, and the full space of model width and reconvolution beamwidth was explored for several algorithms and point spread functions.

\section{The simulations}

All data are noiseless, with no calibration errors. The dirty map was created by direct convolution of the model with the PSF to avoid sampling effects. The uv coverage was that of the A configuration VLA at $8.4 \mathrm{GHz}$ for a source at 33 degrees

* On leave from NRAO 
declination. The image cell size was .03", heavily oversampled. PSFs were uniformly weighted, derived from full 8 hour tracks, a 5 minute snapshot, and an intermediate synthesis of five snapshots.

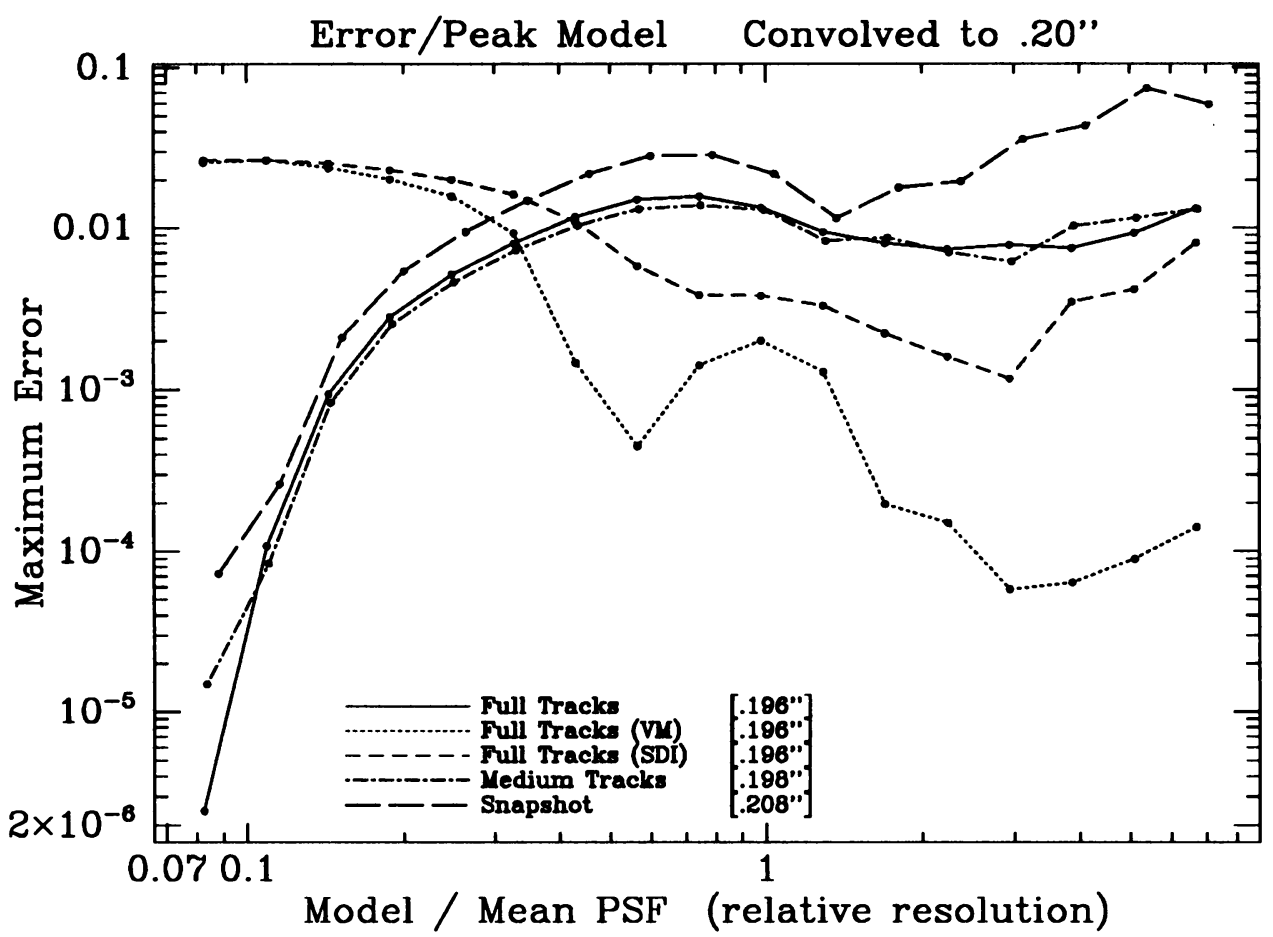

Fig. 1. Maximum error in deconvolutions of a Gaussian source. The PSF corresponding to full track sampling at the VLA has been deconvolved with Högbom CLEAN, SDI-CLEAN, and MEM-VM. The other two PSFs have been deconvolved with Högbom CLEAN. The ordinate is model FWHM divided by .200 ". The fitted FWHM to each PSF is in brackets.

\section{Results}

- Deconvolution errors can be quite significant, even for a well sampled VLA observation. These errors often look like calibration errors, and have probably been misdiagnosed in the past, along with whatever calibration errors are actually present.

- Modelling can be used to demonstrate presence of deconvolution errors

- CLEAN errors are dominated by uv plane extrapolation, not holes in the sampling pattern or lack of zero spacings. This maxes CLEAN based algorithms very amenable to smoothing.

- MEM errors have a significant zero spacing component. MEM images are often significantly better than the CLEAN images at nominal resolution, but cannot be improved by post smoothing. Fractional RMS error may actually get worse with smoothing. 
- The source itself masks the on-source CLEAN errors. This leads to a strong peak in "off-source errors" as a function of source size when the source size is slightly less than the synthesized beamwidth.

- $\quad$ Even very mild resolution produces significant CLEAN deconvolution errors.

- CLEAN is significantly better than MEM or SDI-CLEAN at estimating source fluxes, even for extended sources!

- Either CLEAN or MEM is more effective than SDI-CLEAN for nearly all size scales

\section{Discussion}

It is clear from the curves of error vs. model size that one should carefully select the algorithm that matches the size scale of the problem. MEM is superior in maximum error over a wide range of extended scales, but compact features are common in astronomy and cannot be ignored. Also, MEM images are biased towards Gaussians, (Narayan \& Nityananda 1986), so these results are somewhat more favorable to MEM than with more realistic sources. CLEAN has the weight of tradition behind it and is easier to guide which probably leads to it being used in inappropriate circumstances. A hybrid approach of CLEAN and MEM is superior to anything tested here, but is quite awkward to manipulate with existing tools. Clearly there is need for a good software implementation of a hybrid algorithm.

To a good approximation, the CLEAN errors in the visibility plane are modeled well by a Heavyside function at the outer envelope of uv sampling. This discovery explains the observed smoothing properties. The degree of smoothing adjusts how much of the erroneous extrapolation is included in the final map. Future work will include investigation of optimal non-Gaussian restoring beams to balance the tradeoffs between resolution, fidelity, and map plane aesthetics. Modelling is still the best means of diagnosing deconvolution errors, but this characteristic error pattern in CLEAN should lead to a simple predictive formula for error estimation.

In the worst case of a source with size comparable to the synthesized beam, the maximum off-source CLEAN error can be as large as few times $10^{-3}$. Errors of this magnitude would almost certainly be diagnosed as calibration errors, and yet they are solely due to deconvolution limitations. The error vs. model size curves for the VLA and VLBA are almost identical, even though the VLBA uv sampling is much sparser. This is because both instruments are equally vulnerable to extrapolation. The consequences for the VLBA may be more severe, as the slightly resolved case will be much more common than at the VLA.

\section{References}

Cornwell T.J. and Evans, K.F.: 1985, Astron. Astrophys. 143, 77-83

Högbom J.A.: 1974, Astron. Astrophys. Suppl. Ser. 15, 417-426

Narayan R. and Nityananda R.: 1986, Ann. Rev. Astron. Astrophys. 24, 127-70

Steer D.G., Dewdney, P.E., and Ito, M.R.: 1984, Astron. Astrophys. 137, 159-165

Wakker B.P. and Schwarz U.J.: 1988, Astron. Astrophys. 200, 312-322 